\title{
Forgetting of stimulus attributes: Some implications for hippocampal models of memory
}

\author{
David C. Riccio ${ }^{1}$ and Robin L. Joynes \\ Department of Psychology, Kent State University, Kent, Ohio 44242, USA
}

Historically, research on memory using animal models has focused on recall of the learned target response. For example, do subjects fail to show conditioned fear after a long retention interval or after an amnestic treatment? Do they remember (or not) to make the correct choice in a maze? These tasks have their counterparts, of course, in studies of human memory, where the focus has often been on manipulations affecting the retention of a list of items or other learned materials.

However, another class of memory phenomena has received far less attention, at least in the animal literature. We refer to the memory for the attributes of the context (background stimuli) in which the target response was acquired. It is well known that changing the training stimulus produces a decrement in responding, resulting in a stimulus generalization gradient. Similarly, a number of studies have shown that, when a test is given in a context different from conditioning, performance is impaired (the "context-shift effect"), presumably because some of the retrieval cues are no longer present. Based on these findings, scientists investigating memory for contextual stimuli ask whether changes in the context that initially disrupt performance become less disruptive over time, seen as a broadening or flattening of the contextual gradient. A seminal study by Perkins and Weyant (1958) illustrates the point. Rats trained on a foodrewarded runway task ran more slowly when the context (runway color) was altered 1 min after conditioning, but they ran equally fast in the original and novel contexts after a delay of $1 \mathrm{wk}$. As the authors noted, the rats forgot the characteristics that distinguished the runways faster than the running response itself. A similar phenomenon involving apparatus contexts was soon reported in a fear-motivated task (McAllister and McAllister, 1963). Later, several empirical papers exploring what came to be called the "forgetting of stimulus attributes" of contexts appeared over the years (Feinberg and Riccio, 1990; Zhou and Riccio, 1996; Anderson and Riccio, 2005) as well as conceptual papers elaborating on the implications of the phenomenon (Riccio et al. 1984, 1992, 1994).

The papers by Wiltgen and Silva (2007) and Biedenkapp and Rudy (2007) in Learning and Memory and another by Winocur et al. (2007) provide important contributions to this topic in a number of ways. At a general level, all the papers call attention to the relevance of the forgetting of attributes to the interests of the neuroscience community, as most of the previous research on memory for attributes has been oriented more toward those with largely a behavioral focus. More specifically, these studies begin to address questions about the biological substrates and mechanisms mediating the flattening of the contextual gradient, a topic nearly totally neglected to date.

With respect to empirical contributions, all three studies, using different species and parameters, independently replicate and confirm the basic phenomenon, providing us with clear and

\footnotetext{
'Corresponding author.
}

E-mail driccio@kent.edu; fax (330) 672-3786.

Article is online at http://www.learnmem.org/cgi/doi/10.1101//m.617107. cohesive examples of the role of contextual memory in conditioned fear. These independent laboratories observe a decrease in conditioned fear when subjects are tested in a context that differs from the one that was used during training (the context-shift effect). Moreover, when a delay (retention interval) is interposed between exposure to the training context and test, the contextshift effect is eliminated.

Importantly, the studies investigate the broadening of the gradient in somewhat different ways. In the Biedenkapp and Rudy (2007) report, rats receive footshocks in one context and are then tested in either the training context or a different context after one of several delays. The context shift effect obtained after a 1-d retention interval is no longer obtained after 7 or $15 \mathrm{~d}$.

Using mice rather than rats and conducting the tests in the original or novel environments after 1, 14, 28, or $36 \mathrm{~d}$, Wiltgen and Silva (2007) also find that the context shift effect diminishes as a function of the retention interval. In a second experiment, they use a single relatively brief pre-exposure to the context to establish a memory representation and then vary the time between that pre-exposure and testing ( 1 or $36 \mathrm{~d}$ ). Relative to nonpre-exposed controls, the exposed mice show more freezing during the test trial. However, the context change affects the freezing differentially, depending on the delay condition: At the short retention interval, little generalization between contexts is seen, whereas at the long interval fear is comparable in both contexts. As the interval between conditioning and testing is one day in all cases, the possibility of fear incubation can be ruled out. The experiments of Winocur et al. (2007) employ not only a contextual fear conditioning situation, as do the other studies, but also a food-preference conditioning paradigm with rats. Using retention intervals of $1 \mathrm{~d}$ and 8 or $28 \mathrm{~d}$ (for the food-preference and fear tasks, respectively), they find that testing in a context different from training disrupts performance at the short interval but not at the long interval. In summary, all three groups show that, over time, animals will forget the specific stimulus attributes associated with the training context and begin to show conditioned responding in the presence of other, similar contexts (a broadening of the generalization gradient). This evidence converges with previous studies described above that have demonstrated forgetting of contextual cues over various retention intervals and in a variety of other paradigms.

More importantly, Biedenkapp and Rudy (2007) and Wiltgen and Silva (2007) extend their findings by demonstrating that this forgetting of specific contextual features can be prevented or reversed. Biedenkapp and Rudy (2007) go about this by adding more context pre-exposures before the training in order to establish a stronger contextual representation. Prior to fear conditioning, rats receive three daily exposures to the training context in order to establish a strong representation in memory (controls are exposed to an irrelevant context). Consistent with the view that the broadening of the gradient reflects forgetting that should be modifiable, pre-exposure in the relevant context maintains the context shift effect over the long interval. To the best of our knowledge, this study is the first to show clearly that a ma- 
nipulation prior to training will improve retention of the contextual attributes (cf. Rosas and Bouton 1997). Furthermore, Biedenkapp and Rudy (2007) note that the differential levels of fear in the two contexts after the long interval provides evidence, consistent with the conclusion of Wiltgen and Silva (2007), that the flattening of the gradient cannot be attributed to incubation of fear.

Taking a different approach to improving memory for attributes of context, Wiltgen and Silva (2007) add a reminder after the retention interval, just before testing. This "reminder" treatment, a brief re-exposure to the training context, serves to resharpen the contextual gradient, i.e., the context shift effect is again obtained. As the authors note, a similar outcome has been obtained by Zhou and Riccio (1994) (see also Rosas and Bouton 1997).

Also, as a side note, and consistent with work by Fanselow (1990), the pre-exposures used in the Learning and Memory papers facilitated performance rather than impairing it. From a conditioning perspective, one might have expected the pre-exposure to impair acquisition of the target response, fear. There is extensive literature demonstrating that experience with the predictive signal (conditioned stimulus) prior to training impairs learning and/or performance of the conditioned response across a wide variety of tasks, the so-called "latent inhibition" effect (Lubow and Moore, 1959). However, no latent inhibition appears to have been observed, likely due to their choice of parameters.

While the evidence of the forgetting of contextual features is interesting in and of itself, what makes the studies truly innovative are the attempts to map these behavioral manifestations of memory onto possible brain regions (hippocampus and PFC) and identify a possible neurological mechanism (LTP). Neuroscientists have proposed that, during memory consolidation, hippocampus activation is involved in retrieving newly acquired memories, but over time, storage of information about specific stimulus attributes has been distributed to other neocortical sites. Evidence supporting this idea comes from brain scans performed during memory retrieval that show high hippocampal and low PFC activation for newly acquired memories and the reverse for old memories. Also, hippocampal brain lesions often disrupt memories for recent events while sparing older memories. Biedenkapp and Rudy (2007) acknowledge that brain activation during memory formation shifts as the memory ages, but they propose that this change in activity may be a function of forgetting rather than a function of time alone. They suggest that forgetting increases the difficulty of retrieval processes, leading to the greater activity in the PFC. Because forgetting and the passage of time often co-occur, interpretation of brain scan studies to date is difficult. While the findings presented in their most recent paper do not provide decisive evidence one way or the other, the authors do show that this alternative interpretation is possible. This outcome leads one to wonder whether future PET and fMRI studies will show less shift of activity from hippocampus to PFC in subjects that experience less attribute forgetting? And likewise, if you speed up forgetting, do you see the brain activity shift from hippocampus to PFC more rapidly?

The evidence that the forgetting of contextual attributes can be reversed by a reminder not involving further training leads Wiltgen and Silva (2007) to raise this interesting question: If the memory is transformed over time as the neocortex extracts and stores common features, how is it possible to obtain the original representation by a reminder exposure? One possibility, of course, is that both representations co-exist (see also Winocur et al. 2007), although this would seem to imply that the hippocampus has longer-term involvement with memory than previously thought.
In an attempt to determine somewhat more directly the possible contribution of the hippocampus to memory for stimulus attributes, Wiltgen and Silva (2007) take a genetic approach by adding a group of $\mathrm{H}$-Ras mutant mice-a genetic mutation that leads to enhanced hippocampal LTP. Presumably, if hippocampal activity helps to encode memory for the context, then these mice may be more resistant to the effects of a retention interval. However, after a 36-d delay, H-Ras mutant mice showed fear to both the training and the alternate contexts, indistinguishable from their wild-type counterparts, suggesting that enhanced hippocampal LTP does not impact memories for contextual details. Alternatively, with only two time points to assess retention, it is possible that any advantage for the H-Ras mice might have been obscured by the long 36-d delay; perhaps intermediate delays would show a dissociation between the groups.

Winocur et al. (2007) employ an alternative strategy in which the hippocampus is lesioned to investigate its role in detecting differences in contextual attributes. Using this approach they find that the sensitivity to context shift at the short interval is eliminated by hippocampal lesions, i.e., performance is similar in both the original and new contexts, suggesting that the context sensitivity was mediated by the hippocampus (see also Penick and Solomon 1991). Furthermore, these authors contrast the traditional consolidation view with that of a transformational concept. The former position assumes that memory is held in the hippocampus for a limited time until a veridical representation of the information is stored in the neocortex. In contrast, the latter view implies that the memory is modified into a more schematic (generalized or gist) representation in neocortical structures. They interpret the impaired performance in the shifted context after a short delay followed by contextindependent responding at the long interval in control rats as consistent with the transformational model.

As is often the case, these valuable contributions raise other intriguing questions. For example, is the hippocampus involved when old memories are reactivated? What neurological processes are involved when reminders "sharpen" an old contextual memory, as reflected in the return of the context shift effect? In a related manner, we would call attention to another implication of the forgetting of stimulus characteristics observed in these studies for a different phenomenon, that of retrograde amnesia (RA). Although the typical temporal gradient of RA indicates that older information is not vulnerable to an amnesic agent, there is now substantial evidence of an important exception to this principle: When old memory is reactivated by brief re-exposure to the training cue (CS), the memory can again become susceptible to ECS or other amnesic agents (Misanin et al. 1968; for reviews, see Sara 2000; Riccio et al. 2006). This phenomenon, often referred to as "reconsolidation," was the topic of a special section of Learning and Memory in 2006 (Volume 13, Issue 5). To date, the original training stimulus or context has been used to reactivate the memory. The context shift effect seen here at the short intervals indicates that an altered stimulus would not be effective as a source of reactivation, since the poor performance presumably reflects the lack of retrieval of the memory. However, the increase in functional similarity of contexts over time suggests that a wider range of situations will serve to reactivate memory, making it vulnerable to RA. In short, it should be possible to induce amnesia for old memory when exposure to a different context is given as a reactivation manipulation after a long but not a short retention interval, an issue that our laboratory has recently begun to explore.

\section{References}

Anderson, M.J. and Riccio, D.C. 2005. Ontogenetic forgetting of stimulus attributes. Learn. Behav. 33: 444-453. 
Biedenkapp, J.C. and Rudy, J.W. 2007. Context preexposure prevents forgetting of a contextual fear memory: Implication for regional changes in brain activation patterns associated with recent and remote memory tests. Learn. Mem. 14: 200-203.

Fanselow, M.S. 1990. Factors governing one trial contextual conditioning. Anim. Learn. Behav. 18: 264-270.

Feinberg, G. and Riccio, D.C. 1990. Changes in memory for stimulus attributes: Implications for tests of morphine tolerance. Psychol. Sci. 1: $265-267$.

Lubow, R.E. and Moore, A.U. 1959. Latent inhibition: The effect of nonreinforced preexposure to the conditioned stimulus. J. Comp. Physiol. Psychol. 52: 415-419.

McAllister, W.R. and McAllister, D.E. 1963. Increase over time in the stimulus generalization of acquired fear. J. Exp. Psychol. 65: 576-582.

Misanin, J.R., Miller, R.R., and Lewis, D.J. 1968. Retrograde amnesia produced by electroconvulsive shock after reactivation of a consolidated memory trace. Science 160: 554-555.

Penick, K. and Solomon, P. 1991. Hippocampus, context, and conditioning. Behav. Neurosci. 105: 611-617.

Perkins, C.C. and Weyant, R.G. 1958. The interval between training and test trial as determiner of the slope of generalization gradients. J. Comp. Physiol. Psychol. 51: 596-600.

Riccio, D.C., Richardson, R., and Ebner, D.L. 1984. Memory retrieval deficits based upon altered contextual cues: A paradox. Psychol. Bull. 96: $152-165$.
Riccio, D.C., Ackil, J., and Burch-Vernon, A. 1992. Forgetting of stimulus attributes: Methodological implications for assessing associative phenomena. Psychol. Bull. 112: 433-445.

Riccio, D.C., Rabinowitz, V.C., and Axelrod, A. 1994. Memory: When less is more. Am. Psychol. 49: 917-926.

Riccio, D.C., Millin, P.M., and Bogart, A.R. 2006. Reconsolidation: A brief history, a retrieval view, and some recent issues. Learn. Mem. 13: $536-544$.

Rosas, J.M. and Bouton, M.E. 1997. Additivity of the effects of retention interval and context change on latent inhibition: Toward resolution of the context forgetting paradox. J. Exp. Psychol. Anim. Behav. Process 23: 283-294

Sara, S.J. 2000. Retrieval and reconsolidation: Toward a neurobiology of remembering. Learn. Mem. 7: 73-84.

Wiltgen, B.J. and Silva, A.J. 2007. Memory for context becomes less specific with time. Learn. Mem. 14: 313-317.

Winocur, G., Moscovitch, M., and Sekeres, M. 2007. Memory consolidation or transformation: Context manipulation and hippocampal representations of memory. Nat. Neurosci. 10: 555-557.

Zhou, Y.L. and Riccio, D.C. 1994. Pretest cuing can alleviate the forgetting of contextual stimulus attributes. Learn. Motiv. 25: 233-244.

Zhou, Y.L. and Riccio, D.C. 1996. Manipulation of components of context: The context shift effect and forgetting of stimulus attributes. Learn. Motiv. 27: 400-407. 


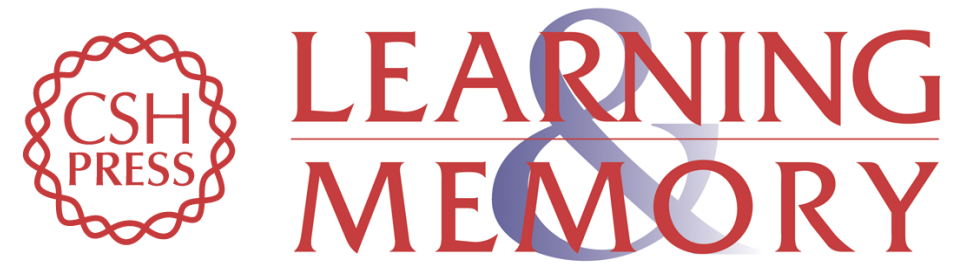

\section{Forgetting of stimulus attributes: Some implications for hippocampal models of memory}

David C. Riccio and Robin L. Joynes

Learn. Mem. 2007, 14:

Access the most recent version at doi:10.1101//m.617107

References This article cites 19 articles, 5 of which can be accessed free at:

http://learnmem.cshlp.org/content/14/6/430.full.html\#ref-list-1

License

Email Alerting Receive free email alerts when new articles cite this article - sign up in the box at the Service top right corner of the article or click here. 